\title{
Global Analysis of Solar Neutrino Oscillations Including SNO CC Measurement
}

\author{
John N. Bahcall \\ School of Natural Sciences, Institute for Advanced Study, Princeton, NJ 08540 \\ E-mail: jnb@ias.edu
}

\author{
M. C. Gonzalez-Garcia \\ Theory Division, CERN, CH-1211, Geneva 23, Switzerland, \\ C.N. Yang Institute for Theoretical Physics \\ State University of New York at Stony Brook \\ Stony Brook, NY 11794-3840, USA, \\ and Instituto de Física Corpuscular, Universitat de València-C.S.I.C. \\ Edificio Institutos de Paterna, Apt 22085, 46071 València, Spain \\ E-mail: concha@thwgs.cern.ch
}

\section{Carlos Peña-Garay \\ Instituto de Física Corpuscular, Universitat de València-C.S.I.C. \\ Edificio Institutos de Paterna, Apt 22085, 46071 València, Spain \\ E-mail: penya@ific.uv.es}

\begin{abstract}
For active and sterile neutrinos, we present the globally allowed solutions for two neutrino oscillations. We include the SNO CC measurement and all other relevant solar neutrino and reactor data. Five active neutrino oscillation solutions (LMA, LOW, SMA, VAC, and Just $\mathrm{So}^{2}$ ) are currently allowed at $3 \sigma$; three sterile neutrino solutions (Just $\mathrm{So}^{2}, \mathrm{SMA}$, and VAC) are allowed at $3 \sigma$. The goodness of fit is satisfactory for all eight solutions. We also investigate the robustness of the allowed solutions by carrying out global analyses with and without: 1) imposing solar model constraints on the ${ }^{8} \mathrm{~B}$ neutrino flux, 2) including the Super-Kamiokande spectral energy distribution and day-night data, 3) including a continuous mixture of active and sterile neutrinos, 4) using an enhanced CC cross section for deuterium (due to radiative corrections), and 5) an optimistic, hypothetical reduction by a factor of three of the error of the SNO CC rate. For every analysis strategy used in this paper, the most favored solutions all involve large mixing angles: LMA, LOW, or VAC. The favored solutions are robust, but the existence at $3 \sigma$ of individual sterile solutions and the active Just $\mathrm{So}^{2}$ solution is sensitive to the analysis assumptions.
\end{abstract}


KEYWORDS: solar and atmospheric neutrinos, neutrino and gamma astronomy, neutrino physics. 


\section{Contents}

1. Introduction 1

2. Calculational Procedures 3

2.1 Definition of $\chi^{2}$ for the global analysis

$2.2 \quad$ A level playing field for sterile neutrinos 5

3. Global Solutions including Rates and Day-Night Spectra

4. Rates-only Global Solutions 12

5. Comparisons and Clarifications 16

5.1 A priori rejecting sterile neutrinos 17

5.2 Delicate differences in the error analysis 17

5.3 Mixtures of sterile and active neutrinos

6. Discussion 20

\section{Introduction}

The epochal Sudbury Neutrino Observatory (SNO) measurement [1] of the CC rate for solar neutrino absorption by deuterium provides an important new constraint on the allowed neutrino oscillation solutions. We present in this paper solutions for the globally allowed regions that include, in addition to the SNO CC measurement, the available data from the Chlorine [2], Gallium [3, 4, 5], and Super-Kamiokande [6] experiments. We emphasize tests of the robustness of the global solutions to different analysis strategies.

The most radical test we make is to determine the allowed solutions if only the total rates of the solar neutrino experiments are used, ignoring the beautiful data from the Super-Kamiokande measurements of the spectral energy distribution and the day-night variations. This test is motivated by the fact that the SuperKamiokande day and night recoil energy spectra provide 38 different data points, while the rates of the chlorine experiment, the two gallium experiments, and SNO provide together only 4 data points. Some authors have questioned whether $\chi^{2}$ fits to the complete data set overweight the Super-Kamiokande data because of the large number of spectral energy bins [7]. 
We take both sides of the somewhat philosophical question of whether the ${ }^{8} \mathrm{~B}$ neutrino flux should be allowed to vary without considering constraints from the solar model. We find self-consistent global solutions both with and without imposing the solar model constraints.

We require (cf. [8]) that the ${ }^{8} \mathrm{~B}$ neutrino flux be treated the same way in all aspects of the calculation, either completely free when evaluating both the rates and the energy spectrum or constrained everywhere by the solar model predictions. We also require that the ${ }^{8} \mathrm{~B}$ neutrino flux be identical in all parts of the calculation. Finally, we avoid double counting the Super-Kamiokande rate measurement; we do not include the measured flux normalization both in the rate measurement and in the spectral energy distribution.

We treat sterile neutrinos on the same basis as active neutrinos, which implies that our $\chi^{2}$ plots are made in terms of three free parameters : $\Delta m^{2}, \tan ^{2} \theta$, and a third parameter, $\cos ^{2} \eta$, which defines the active-sterile admixture. We carry out calculations for a variety of values of $\cos ^{2} \eta$, but, with one exception that is discussed in Section 4 and Section 5.3, the absolute minimum in $\chi^{2}$ always lies in the active neutrino plane. We also describe how the allowed regions are affected if one rules out a priori the possibility that sterile neutrinos exist and therefore constructs the allowed regions in $\chi^{2}$ using, as has usually been done in the past, only two free parameters: $\Delta m^{2}$ and $\tan ^{2} \theta$.

Why do all these tests? There are not yet enough solar neutrino experiments to ensure cross checks and redundancy in the data. Therefore, different plausible analysis schemes can lead to different conclusions. The existence of a particular allowed region, e. g., the SMA solution or the Just $\mathrm{So}^{2}$ solution, may depend upon which of several possible plausible analysis schemes are used. Our motto is: "If its not robust, its not believable."

In section 2, we summarize the calculational procedures. Because of the excellent agreement between the predicted standard solar model flux of ${ }^{8} \mathrm{~B}$ neutrinos (see ref. [9], hereafter BP00) and the combined SNO [1] and Super-Kamiokande [6] measurement, we include the theoretical fluxes and their BP00 uncertainties in our standard analysis. We also use a 'level playing field' prescription for evaluating the allowed active and sterile neutrino solutions. We present in section 3 the allowed solutions that exist if all the solar neutrino data, including the spectral energy distribution and day night data of Super-Kamiokande, are included in the analysis. Considering only the total rates in the chlorine, gallium, Super-Kamiokande, and SNO experiments, we present in section 4 the allowed solutions for this extreme case. We also investigate the effects of allowing the ${ }^{8} \mathrm{~B}$ flux to vary unconstrained by solar model predictions, of using a larger CC cross section for deuterium (motivated by possible effects of radiative corrections [10]), and of a hypothetical reduction by a factor of three in the total quoted error for the SNO CC rate. In section 5, we discuss how some different approaches to the analysis of solar neutrino data affect the 
allowed regions. We pay particular attention in this section to the a priori rejection of sterile neutrinos, the role of the measured ${ }^{8} \mathrm{~B}$ neutrino flux normalization and the influence of the standard solar model neutrino flux constraint, and the influence of the day-night spectral energy data on the allowed sterile neutrino component. We summarize our results in section 6 .

\section{Calculational Procedures}

This section is intended primarily for aficionados of neutrino oscillation analyses.

We use, unless stated otherwise, the techniques and parameters for the analysis that we have described elsewhere [8] and [11]. In ref. [8], from which we derive our primary analysis strategy, the focus was on allowed solutions in which the ${ }^{8} \mathrm{~B}$ flux was unconstrained by any solar model considerations. In this paper, we derive and contrast global solutions in which the ${ }^{8} \mathrm{~B}$ flux is constrained, or unconstrained, by the standard solar model uncertainty.

We first describe in section 2.1 the procedure we use for calculating $\chi^{2}$ for the global analysis and then discuss in section 2.2 the impartial manner in which we treat active and sterile neutrinos.

\subsection{Definition of $\chi^{2}$ for the global analysis}

In section 3, we determine the allowed range of the oscillation parameters using the $\mathrm{CC}$ event rate measured at SNO, the Chlorine and Gallium event rates (we use here the weighted averaged GALLEX/GNO and SAGE rates), and the $2 \times 19$ bins of the (1258 day) Super-Kamiokande electron recoil energy spectrum measured separately during the day and night periods. In this global analysis we adopt the prescription described in ref. [8]. We do not include here the Super-Kamiokande total rate, since to a large extent the total rate is represented by the flux in each of the spectral energy bins. We define the $\chi^{2}$ function for the global analysis as:

$$
\chi_{\text {global }}^{2}=\sum_{i, j=1,41}\left(R_{i}^{t h}-R_{i}^{e x p}\right) \sigma_{G, i j}^{-2}\left(R_{j}^{t h}-R_{j}^{e x p}\right),
$$

where $\sigma_{G, i j}^{2}=\sigma_{R, i j}^{2}+\sigma_{S p, i j}^{2}$. Here $\sigma_{R, i j}$ is the corresponding $41 \times 41$ error matrix containing the theoretical as well as the experimental statistical and systematic uncorrelated errors for the 41 rates while $\sigma_{S p, i j}$ contains the assumed fully-correlated systematic errors for the $38 \times 38$ submatrix corresponding to the Super-Kamiokande day-night spectrum data. We include here the energy independent systematic error which is usually quoted as part of the systematic error of the total rate. The error matrix $\sigma_{R, i j}$ includes important correlations arising from the theoretical errors of the solar neutrino fluxes, or equivalently of the solar model parameters.

When considering just the total rates (see section (4), we adapt the $\chi^{2}$ definition of ref. [12, 13] to include the two different gallium rates and the new SNO CC event 
rate. The $\chi^{2}$ for this case is

$$
\chi_{\text {Rates }}^{2}=\sum_{i, j=1,5}\left(R_{i}^{t h}-R_{i}^{e x p}\right) \sigma_{R, i j}^{-2}\left(R_{j}^{t h}-R_{j}^{e x p}\right)
$$

where $R_{i}^{t h}$ is the theoretical prediction of the event rate in detector $i$ and $R_{i}^{\exp }$ is the measured rate. The error matrix $\sigma_{i j}$ contains the experimental errors, both systematic and statistical, as well as the theoretical uncertainties on the solar neutrino fluxes and the interaction cross sections. The theoretical error matrix includes important correlations arising from the theoretical errors of the solar neutrino fluxes, or equivalently of the solar model parameters. Furthermore for the GALLEX/GNO and SAGE rates, the corresponding theoretical errors for the interaction cross section are assumed to be fully correlated. In our statistical treatment of the rate data, we adapt the analysis of ref. [12], with the updated uncertainties and distributions for neutrino production fractions and the solar matter density given in ref. [9] and tabulated in http:www.sns.ias.edu/ jnb .

We also present the results of an analysis performed with an unconstrained ${ }^{8} \mathrm{~B}$ neutrino flux. In this analysis, the $\mathrm{SSM}^{8} \mathrm{~B}$ neutrino flux is multiplied by a factor of $f_{\mathrm{B}}$, which is fit to the data. We minimize $\chi^{2}$ with respect to $f_{\mathrm{B}}$ for each set of neutrino oscillation parameters; no theoretical error is included for the ${ }^{8} \mathrm{~B}$ neutrino flux in the error matrix. We restrict ourselves to the range $f_{\mathrm{B}}<2$. We have checked that there are no allowed solutions for values of $f_{\mathrm{B}}>2$ if the Super-Kamiokande day-night recoil energy spectra are included. In the minimization procedure, the factor $f_{\mathrm{B}}$ is required to be the same for the normalization of each Super-Kamiokande spectral energy bin, the SNO CC rate, and the ${ }^{8} \mathrm{~B}$ contributions to the gallium and chlorine experiments.

One of the largest systematic uncertainties in interpreting the SNO CC measurement results from the uncertainty in the absolute value of the neutrino absorption cross section on deuterium [1, 14. We have used in our calculations the cross sections calculated by Nakamura et al. [15], which are in good agreement with the results of Butler et al. [16]. Most recently, Beacomand Parke [10] have argued that there may be a $6 \%$ increase in the cross sections evaluated by Nakamura et al and Butler et al. due to a combination of factors represented by a $2 \%$ correction to $g_{A}^{2}$, and two other factors related to radiative corrections [17]. We follow the SNO collaboration [1, 18] in including the $2 \%$ correction from $g_{A}^{2}$ in our standard calculations.

In order to test the sensitivity of the global fits to the effects of radiative corrections, we have repeated all of our calculations with a CC cross section for SNO that is increased by an additional $4 \%$ relative to the standard cross sections. We shall describe these calculations in the text as having been done with the 'enhanced CC cross sections."

For the SNO CC calculation, we have used the resolution function given in ref. [四], which is slightly broader than we have assumed in our previous analyses. 
Following Fogli, Lisi, and Montanino [19 and de Gouvea, Friedland, and $\mathrm{Mu}-$ rayama [20], we present our results in terms of $\tan ^{2} \theta$ rather than $\sin ^{2} 2 \theta$ in order to include solutions with mixing angles greater than $\pi / 4$ (the so-called 'dark side').

\subsection{A level playing field for sterile neutrinos}

Once the SNO results are included in the analysis, all of the solutions with sterile neutrinos are relatively poor fits to the totality of solar neutrino data. Many different regions of the sterile neutrino parameter space provide comparable fits to the available data. We have therefore made one significant departure from previous analysis techniques for two neutrino oscillations: we treat the active and sterile neutrinos as different aspects of the same two-neutrino oscillation scheme. This procedure 'levels the playing field' for active and sterile neutrinos. The assumption of oscillation into either all active or all sterile neutrinos describes the limiting extremes of a continuum in which the oscillation occurs into a linear combination of active and sterile neutrinos, see discussion in ref. [21] of four neutrino oscillations.

Our theoretical framework contains three free parameters: $\Delta m^{2}, \tan ^{2} \theta$, and the third parameter, $\cos ^{2} \eta$, which defines the active-sterile admixture. We focus here only on the two limiting cases, $\cos ^{2} \eta=1,0$. Since the parameter space is threedimensional, the allowed regions for a given C.L. are defined as the set of points satisfying the condition

$$
\chi_{\mathrm{sol}}^{2}\left(\Delta m^{2}, \theta, \eta\right)-\chi_{\mathrm{sol}, \min }^{2} \leq \Delta \chi^{2} \text { (C.L., } 3 \text { d.o.f.) },
$$

where $\Delta \chi^{2}$ (C.L., 3 d.o.f. $)=6.25,7.81,11.34$, and 14.16 for C.L. $=90 \%, 95 \%$, $99 \%$ and $99.73 \%(3 \sigma)$ respectively, and $\chi_{\text {sol,min }}^{2}$ is the global minimum in the threedimensional space. A similar procedure is used in searching for allowed solutions for three and four neutrino oscillations [21, 22, [19, 12].

If one chooses to ignore the possibility of sterile neutrinos, then one should use in eq.. (2.3) $\Delta \chi^{2}$ (C.L., 2 d.o.f.) instead of $\Delta \chi^{2}$ (C.L., 3 d.o.f.). The corresponding numbers for 2 d.o.f are $\Delta \chi^{2}$ (C.L., 2 d.o.f. $)=4.61,5.99,9.21$, and 11.83 for C.L. $=$ $90 \%, 95 \%, 99 \%$ and $99.73 \%(3 \sigma)$, For the case in which only active neutrinos are considered, the numbers for $\chi_{\min }^{2}$ given in the various tables in this paper can be used together with the above values of $\Delta \chi^{2}$ (C.L., 2 d.o.f.) to determine the smaller allowed regions.

\section{Global Solutions including Rates and Day-Night Spectra}

We describe in this section global solutions obtained by using all the relevant solar neutrino and reactor data.

Figure 1 shows the globally allowed solutions when the Super-Kamiokande recoil energy spectrum during the day and, separately, the energy spectrum at night are 


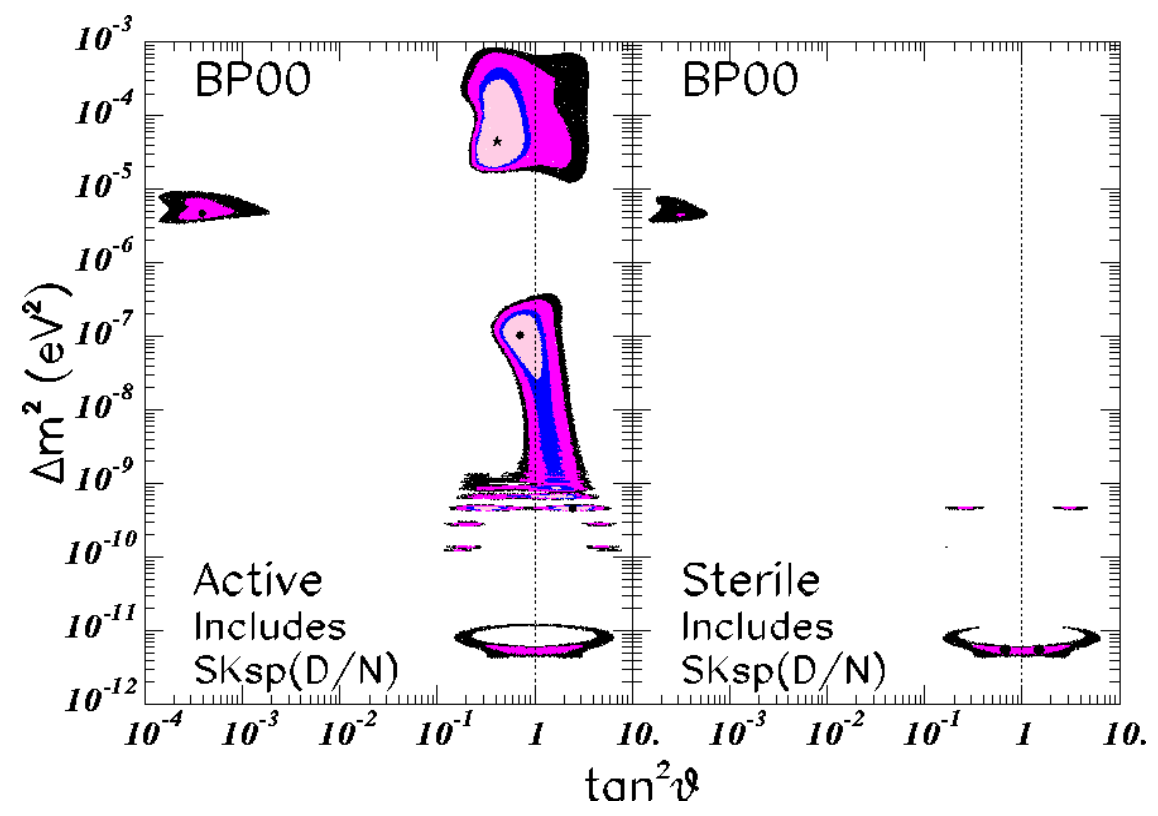

Figure 1: Global solutions including all available solar neutrino data. The input data include the total rates from the SNO [1], Chlorine [2], and Gallium (averaged) [3, 5, 4] experiments, as well as the recoil electron energy spectrum measured by Super-Kamiokande [6] during the day and separately the energy spectrum measured at night. The C.L. contours shown in the figure are $90 \%, 95 \%, 99 \%$, and $99.73 \%(3 \sigma)$. The allowed regions are limited by the Chooz reactor measurements 23] to mass values below $\sim(7-8) \times 10^{-4} \mathrm{eV}^{2}$. The local best-fit points are marked by dark circles. The theoretical errors for the BP00 neutrino fluxes are included in the analysis.

included in addition to the total rates in the Chlorine [2], Gallium (averaged) [3, 旬, 国, and SNO [1] experiments. In order to avoid double counting the Super-Kamiokande total rate(cf. ref. [8] for a discussion of this point), we have not included the SuperKamiokande rate [6] in addition to the Super-Kamiokande day and night energy spectra, which each contain their own absolute normalizations. We have used the Chooz reactor bound [23] to limit the allowed solutions to mass values below $\sim(7-8)$ $\times 10^{-4} \mathrm{eV}^{2}$.

Figure 1 is our currently preferred global solution.

All eight of the allowed solutions for active and sterile neutrinos that existed before the SNO CC measurement (e.g., ref. [8]) are still allowed at $3 \sigma$ after including the results of the SNO CC measurement.

Table 1 gives the best-fit parameters for each of the eight allowed oscillation solutions illustrated in figure 1. We give in table 1 the values of $\Delta m^{2}, \tan ^{2} \theta, \chi_{\min }^{2}$, and the goodness-of-fit for each of the best-fit points. The solutions described by the acronyms like LMA and Just $\mathrm{So}^{2}$ can be identified in figure 1 with the aid of table 1 by using the association between the solution acronyms (column 1) and the values 


\begin{tabular}{lcccc}
\hline Solution & $\Delta m^{2}$ & $\tan ^{2}(\theta)$ & $\chi_{\min }^{2}$ & g.o.f. \\
\hline LMA & $4.5 \times 10^{-5}$ & $4.1 \times 10^{-1}$ & 35.3 & $59 \%$ \\
LOW & $1.0 \times 10^{-7}$ & $7.1 \times 10^{-1}$ & 38.4 & $45 \%$ \\
VAC & $4.6 \times 10^{-10}$ & $2.4 \times 10^{0}$ & 39.0 & $42 \%$ \\
SMA & $4.7 \times 10^{-6}$ & $3.9 \times 10^{-4}$ & 45.4 & $19 \%$ \\
Just So $^{2}$ & $5.5 \times 10^{-12}$ & $0.67(1.5) \times 10^{0}$ & 45.7 & $18 \%$ \\
Sterile Just So $^{2}$ & $5.5 \times 10^{-12}$ & $0.67(1.5) \times 10^{0}$ & 45.8 & $18 \%$ \\
Sterile SMA & $4.5 \times 10^{-6}$ & $3.1 \times 10^{-4}$ & 46.6 & $16 \%$ \\
Sterile VAC & $4.7 \times 10^{-10}$ & $2.7 \times 10^{-1}$ & 47.2 & $15 \%$ \\
\hline
\end{tabular}

Table 1: Best-fit global oscillation parameters with all solar neutrino data. This table corresponds to the global solution illustrated in figure 1. The differences of the squared masses are given in $\mathrm{eV}^{2}$. The number of degrees of freedom is 38 [38(spectrum) +3 (rates) -3 (parameters: $\Delta \mathrm{m}^{2}, \theta$, active-sterile admixture)]. The goodness-of-fit given in the last column is calculated using the value of $\chi^{2} /$ d.o.f for each allowed solution. The BP00 best-fit fluxes and their estimated errors have been included in the analysis. The rates from the GALLEX/GNO and SAGE experiments have been averaged to provide a unique data point. The goodness-of-fit given in the last column is calculated from the value of $\chi^{2} /$ d.o.f at each local minimum (i. e., for LMA, SMA, VAC, LOW, etc.).

of $\Delta m^{2}$ (column 2).

The LMA active solution is the best-fit, but is only slightly better than the LOW and VAC active solutions. The SMA solution is a significantly less good fit than the LMA and LOW solutions. The goodness-of-fit ranges from $59 \%$ for the LMA active solution to $15 \%$ for the Sterile VAC solution, all satisfactory fits to the available data.

The five active solutions, LMA, SMA, LOW, VAC, and Just $\mathrm{So}^{2}$ all appear clearly in the left hand panel of figure 1, although the allowed area of the SMA solution has shrunk significantly relative to the pre-SNO situation (cf. refs. [8, 11]). ${ }^{1}$ The largest reduction in allowed area occurs for the Sterile SMA, which is barely visible at $3 \sigma$ C.L. in the right hand panel of figure 1. The other sterile solutions are not much affected at $3 \sigma$ by the SNO CC measurement, although they have also become somewhat less likely. The Just $\mathrm{So}^{2}$ solution for both active and sterile oscilaltions has also worsened after the inclusion of the SNO CC rate.

For oscillations among only active neutrinos, the statistical criteria used to define the allowed regions are not unique. One could assume that there are no sterile

\footnotetext{
${ }^{1}$ We caution the reader that the comparison with previous results is not entirely straightforward since we have included in the $\chi^{2}$ analysis an additional parameter $(\cos \eta=1,0$, for active or sterile neutrinos) and we measure all departures from $\chi_{\min }^{2}$, the best-fit value with either active or sterile neutrinos. We have also made a few minor adjustments in the analysis code.
} 


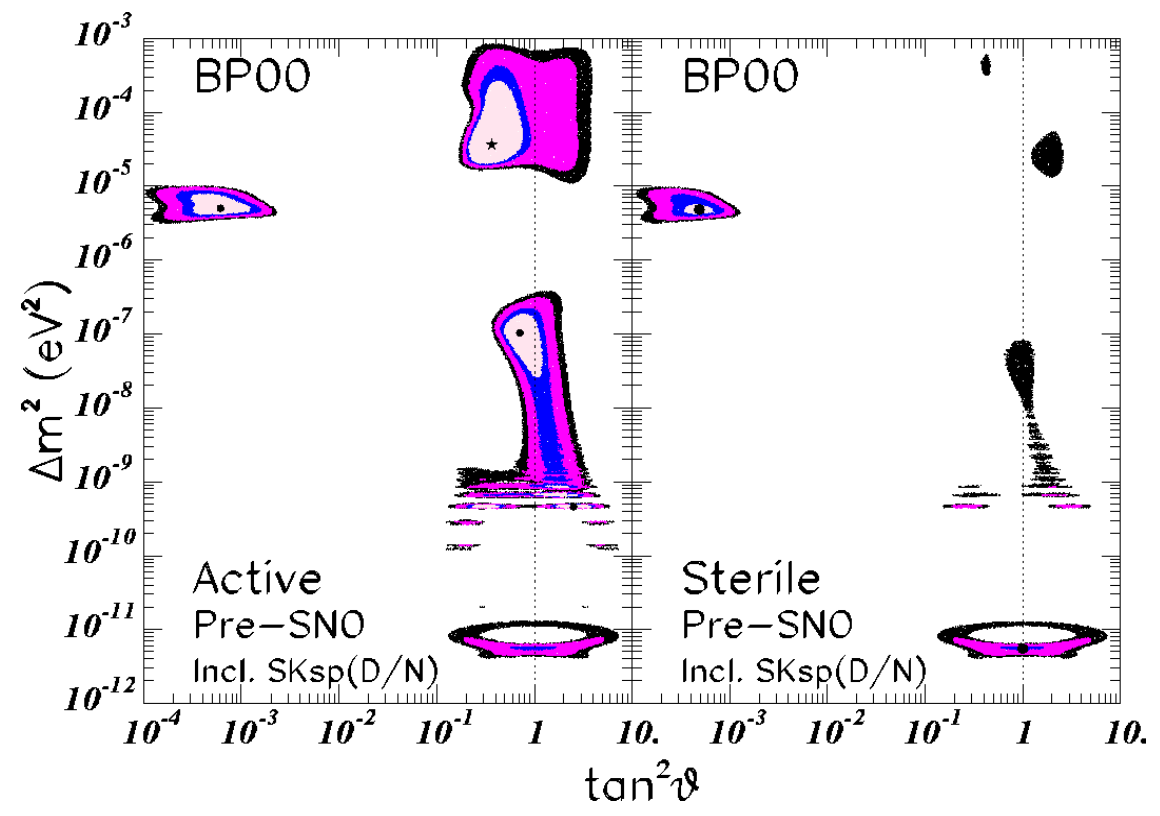

Figure 2: Pre-SNO global solutions. The input data and the analysis procedures are the same as used in producing figure 1, except that we have not included the SNO CC measurement in constructing figure 2 .

neutrinos (one cannot assume that are no active neutrinos.). If one knows a priori that Nature does not contain light sterile neutrinos, then we live in the slice of solution space corresponding to $\cos ^{2} \eta=1$. Given this a priori knowledge, the regions at a given C.L. would be 2-dimensional and then the analysis for the active neutrino oscillations would be the standard one with regions at a given C.L. defined in terms of $\Delta \chi^{2}$ (C.L., 2 d.o.f.) (see discussion in section 2 following eq. 2.3). In this approach, the active SMA region would appear only at $3 \sigma$ since $\chi_{\text {SMA }}^{2}-\chi_{\text {LMA }}^{2}=10.1$.

We discuss explicitly in section 5 (see especially figure 9) the allowed solutions in the case in which one knows, by some other means, that sterile neutrinos do not exist.

How much has the measurement by SNO of the CC rate affected the globally allowed solutions? Figure [1] and figure \&, when compared, answer this question.

The SNO measurement has not changed qualitatively the allowed solution space for active neutrinos. Nevertheless, the SMA solution is now less likely since it is difficult for SMA to fit simultaneously the Super-Kamiokande flat spectrum and the SNO CC measurement. The comparison between the SNO CC measurement and the Super-Kamiokande total rate indicates the presence of some NC contribution to the measured Super-Kamiokande rate. For the SMA solution, the neutral current contribution is only predicted to be large enough for the larger mixing angles. For the larger SMA mixing angles, the survival probability of ${ }^{8} \mathrm{~B}$ neutrinos rises with 


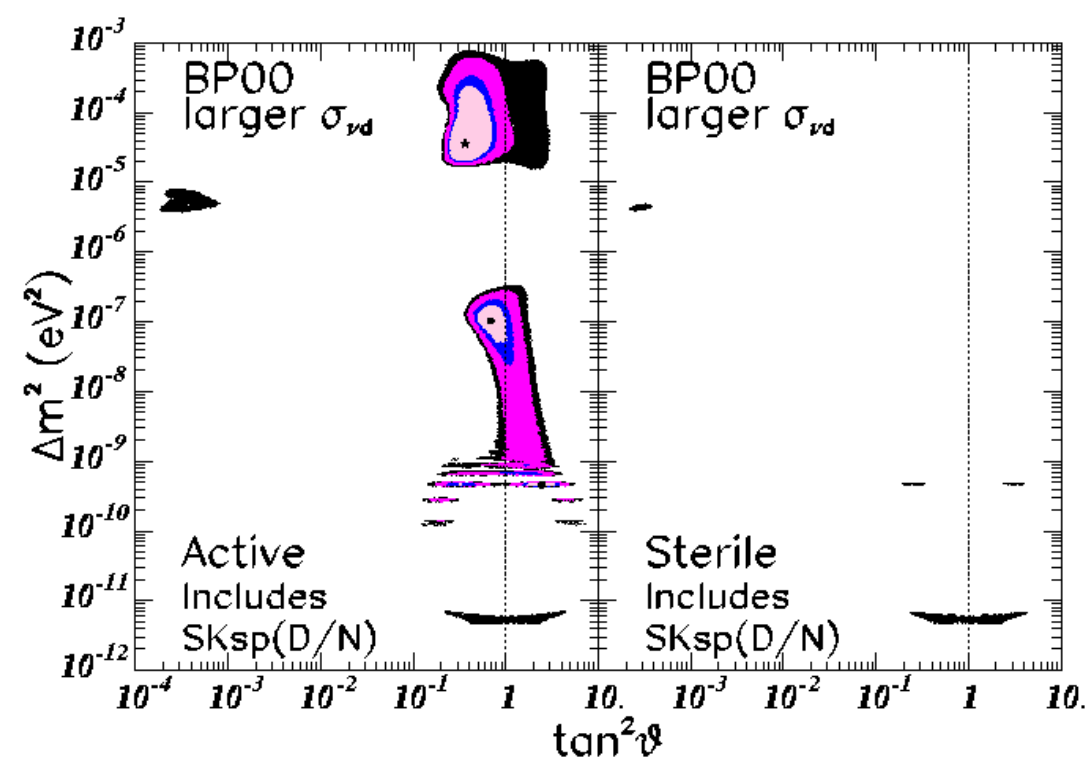

Figure 3: Global solutions including all available solar neutrino data, with enhanced CC cross section for deuterium. The input date are the same as in figure 1 except that we have used a $4 \%$ large CC cross section for neutrino absorption on deuterium.

energy (for this reason the SMA region in figure 6 for the rates-only analysis is shifted towards larger mixing angles). However, this predicted growth of the survival probability with energy conflicts with the Super-Kamiokande measurement [6] that is consistent with an undistorted recoil energy spectrum.

The evidence for a NC contribution in the Super-Kamiokande rate also disfavors oscillations into sterile neutrinos. The LMA and LOW sterile neutrino solutions, previously allowed at $3 \sigma$ are now disfavored at this C.L. and the allowed region of the SMA sterile solution has been reduced in size. The fit is also worsened for the Just $\mathrm{So}^{2}$ solution (from $\chi_{\mathrm{Just} \mathrm{So}^{2}}^{2}-\chi_{\mathrm{LMA}}^{2}=7.6$ in the pre-SNO analysis to $\chi_{\mathrm{Just} \mathrm{So}^{2}}^{2}-\chi_{\mathrm{LMA}}^{2}=$ 10.4 in the post-SNO analysis). We will return to this point in Sec. 5.2 .

What is the effect of the possibly enhanced cross section discussed in ref. 10 and in section 22? Figure 3 and table 2 show the results that are obtained when a CC cross section enhanced by $4 \%$ is assumed for deuterium. It is instructive to compare directly figure 3 and table 8 with figure 1 and table 1. Doing so, we see that no qualitative changes are induced by using the larger CC cross section for deuterium. The main quantitative change is that the allowed regions for the SMA solutions, both active and sterile, become slightly smaller and less statistically likely when the larger $\mathrm{CC}$ cross section is assumed. All of the sterile neutrino solutions become slightly less good fits.

The wonderful measurement of the $\nu_{e}$ flux of ${ }^{8} \mathrm{~B}$ neutrinos that has recently been 


\begin{tabular}{lcccc}
\hline Solution & $\Delta m^{2}$ & $\tan ^{2}(\theta)$ & $\chi_{\min }^{2}$ & g.o.f. \\
\hline LMA & $3.7 \times 10^{-5}$ & $3.7 \times 10^{-1}$ & 34.7 & $62 \%$ \\
LOW & $1.0 \times 10^{-7}$ & $6.9 \times 10^{-1}$ & 39.2 & $42 \%$ \\
VAC & $4.6 \times 10^{-10}$ & $2.4 \times 10^{0}$ & 39.7 & $39 \%$ \\
SMA & $4.6 \times 10^{-6}$ & $3.4 \times 10^{-4}$ & 47.0 & $15 \%$ \\
Just So $^{2}$ & $5.5 \times 10^{-12}$ & $0.67(1.5) \times 10^{0}$ & 46.8 & $15 \%$ \\
Sterile Just So $^{2}$ & $5.5 \times 10^{-12}$ & $0.67(1.5) \times 10^{0}$ & 47.0 & $15 \%$ \\
Sterile SMA & $4.5 \times 10^{-6}$ & $2.9 \times 10^{-4}$ & 48.2 & $12 \%$ \\
Sterile VAC & $4.7 \times 10^{-10}$ & $2.7 \times 10^{-1}$ & 48.5 & $12 \%$ \\
\hline
\end{tabular}

Table 2: Best-fit global oscillation parameters with an enhanced CC cross section for deuterium, corresponding to figure 3. The input data used in constructing this table were the same as were used in constructing table 1 except that for this table we adopted a $4 \%$ larger CC cross section for deuterium.

reported by SNO [1] is the first quantitative result reported by this collaboration. It is therefore plausible that the error on the $\nu_{e}$ flux will decrease with time as the systematic uncertainties become better understood and the statistical errors are reduced by counting more events. In an uncontrolled burst of optimism, we have hypothesized that the quoted experimental error on the $\nu_{e}$ flux will be ultimately reduced by a factor of three while the best-estimate value for the $\nu_{e}$ flux will be unchanged.

Figure 4 shows the effect on the globally allowed solutions of reducing the total error on the CC flux measurement of SNO by a factor of three relative to the total

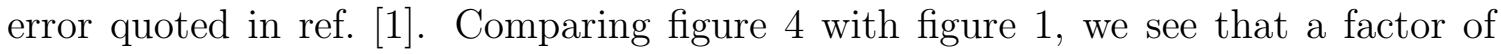
three improvement in the quoted error could eliminate at $3 \sigma$ both the active and the sterile SMA solutions and disfavor (rule out at $3 \sigma$ ) the active (sterile) Just $\mathrm{So}^{2}$ solution. The allowed LMA and LOW solutions would not be much affected by even a factor of three reduction in the error in the $\nu_{e}$ flux, the main effect being a modest reduction of the area of the allowed LMA solution in the $\Delta m^{2}-\tan ^{2} \theta$ plane.

Does it make a difference if we constrain the ${ }^{8} \mathrm{~B}$ neutrino flux according to the predictions of the standard solar model? This is an important question to answer, since one can give reasonable arguments on both sides of the question as to whether or not it is more appropriate to constrain the ${ }^{8} \mathrm{~B}$ neutrino flux. Arguing in favor, one can point out that the standard solar model provides relevant information about the solar interior and about the rates of solar fusion reactions. Moreover, the standard solar model is remarkably successful in predicting results in agreement with helioseismological measurements [9]. On the other hand, we would like to determine the ${ }^{8} \mathrm{~B}$ neutrino flux independent of solar model considerations. For a model-independent analysis, we must allow the ${ }^{8} \mathrm{~B}$ neutrino flux to vary freely. 


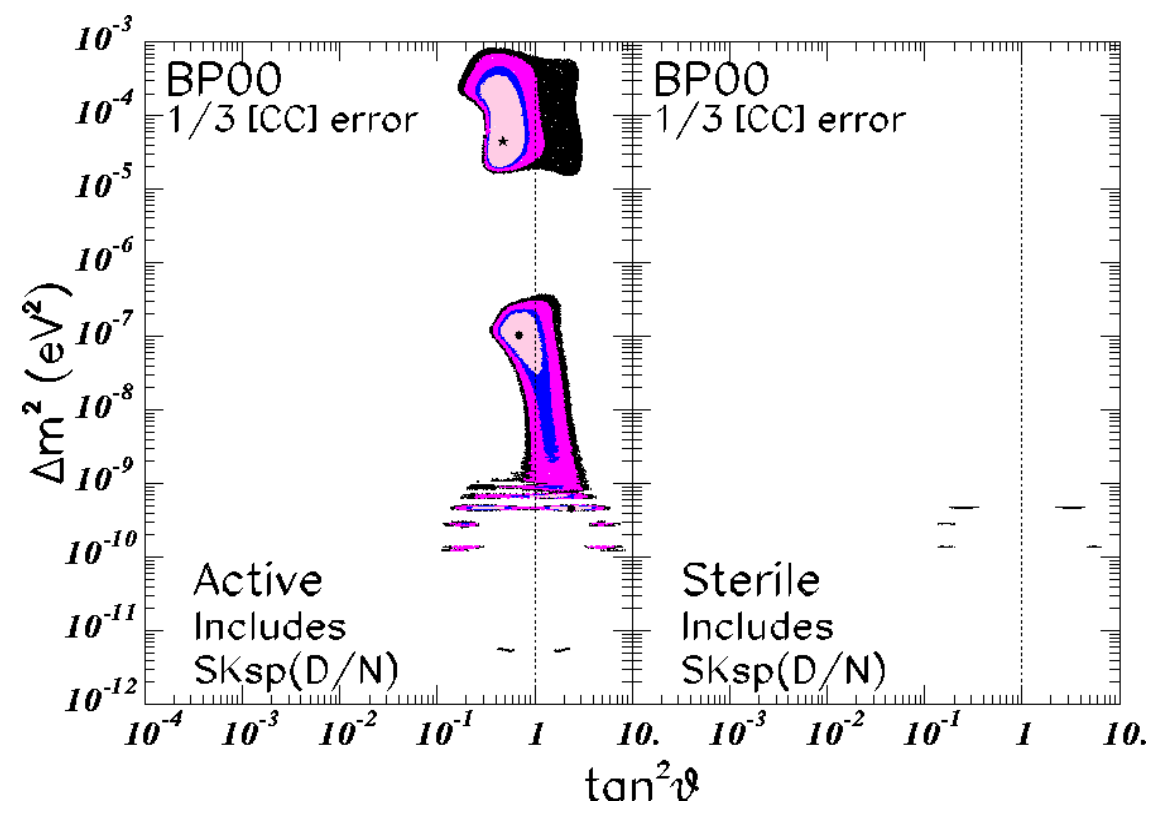

Figure 4: Global solutions with error on SNO CC flux reduced by a factor of three. The input data are the same as for figure 1 except that the error on the $\nu_{e}$ flux of ${ }^{8} \mathrm{~B}$ neutrinos for SNO was artificially reduced by a factor of three.

Figure 5 shows the result of an analysis that is identical to our standard analysis (cf. figure (1) except that in constructing figure 5 the ${ }^{8} \mathrm{~B}$ neutrino flux was not constrained by solar model predictions. We have checked that the global minimum in this case lies in the LMA region for purely active neutrinos, as is also the case for the analysis with the constrained $\mathrm{BP} 00{ }^{8} \mathrm{~B}$ neutrino flux. For the rates-only analysis discussed in the next section, the $\chi^{2}$ minimum for the unconstrained ${ }^{8} \mathrm{~B}$ neutrino flux analysis has non-vanishing components of both active and sterile neutrinos, in agreement with the results of ref. [24] (see also the discussion in Sec. 5).

The three most favored solutions in figure 5 all involve large mixing angles; they are (with their associated g.o.f): LMA (59\%), LOW (39\%), and VAC (35\%). The g.o.f. for all the other solutions is less than $20 \%$.

Comparing figure 5 and figure [1, we see that the favored, large angle solutions, LMA and LOW, are not changed significantly. However, the allowed regions for the SMA and Just $\mathrm{So}^{2}$ solutions, both active and sterile, are reduced in size by performing a ${ }^{8} \mathrm{~B}$-free analysis. The sterile SMA allowed region is reduced to almost a point in figure 5. To find the sterile SMA in figure 5, one has to know where to look, namely, near the $\Delta m^{2}$ and $\tan ^{2} \theta$ for which the best-fit SMA solution appears for active neutrinos. Also, the small sterile VAC region that appears in figure 1 disappears entirely in figure 5 .

It is difficult to anticipate intuitively the quantitative difference between global 


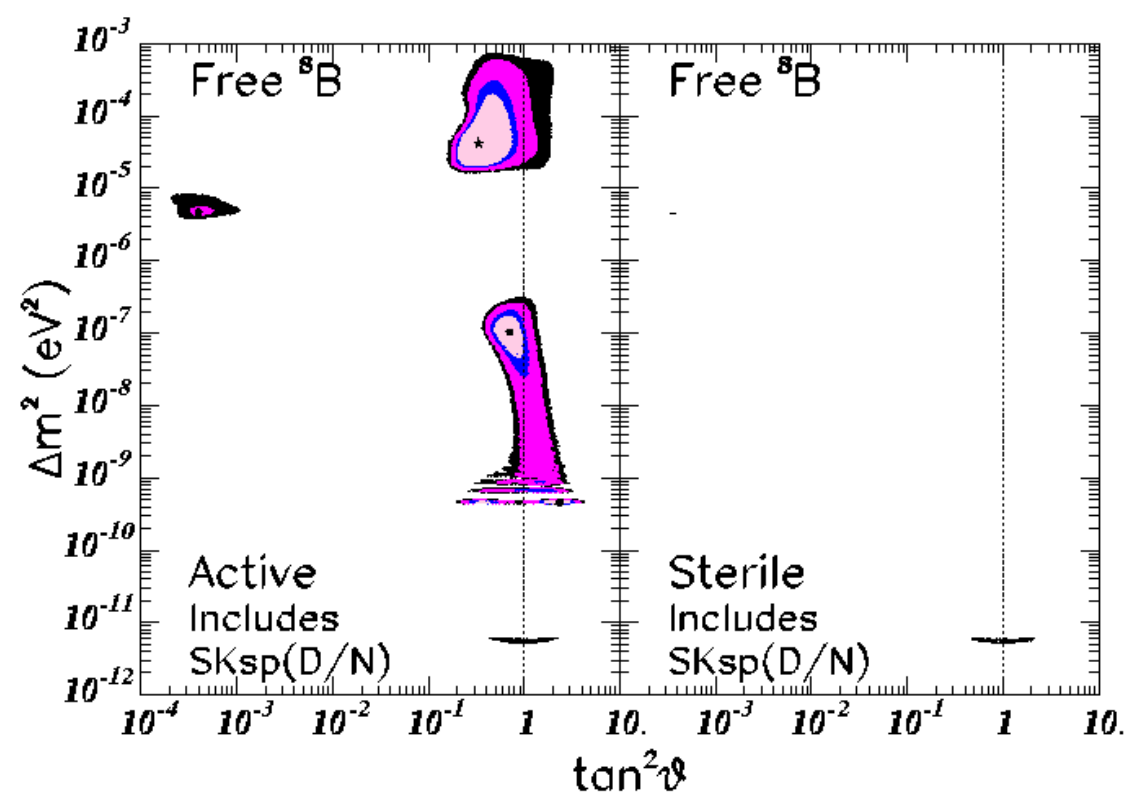

Figure 5: Global solutions, with unconstrained ${ }^{8} \mathbf{B}$ neutrino flux, including all available solar neutrino data. The input data and the analysis are the same as were used in constructing figure 1 except that for the present figure no constraint was placed on the ${ }^{8} \mathrm{~B}$ neutrino flux.

solutions obtained without and without constraints on the ${ }^{8} \mathrm{~B}$ neutrino flux. This is particularly true for the less robust solutions, like all of the sterile solutions and the Just $\mathrm{So}^{2}$ active solution. Two factors work in opposite directions in determining $\chi^{2}$ : 1) the free normalization in the ${ }^{8} \mathrm{~B}$ neutrino flux; and 2) the removal of the theoretical error in the flux. The second effect seems to be generally more important.

\section{Rates-only Global Solutions}

We describe in this section global solutions obtained by considering only the total rates in the Chlorine, GALLEX/GNO, SAGE, Super-Kamiokande, and SNO (CC) solar neutrino experiments. We also evaluate the effects of varying the ${ }^{8} \mathrm{~B}$ neutrino flux unconstrained by solar model predictions and of increasing the $\mathrm{CC}$ cross section for SNO.

Figure 6 and table 3 present the allowed solution regions and the best-fit parameters for the global analysis of only the total rates. The best-fit point is in the VAC region with the LMA solution giving a rather similar $\chi_{\min }^{2}$.

For the LMA, SMA, and LOW solutions, the results appear superficially similar to what is obtained when the Super-Kamiokande spectral data for both the day and the night are included in the analysis (cf. figure 1 and figure 6). However, the g.o.f. 


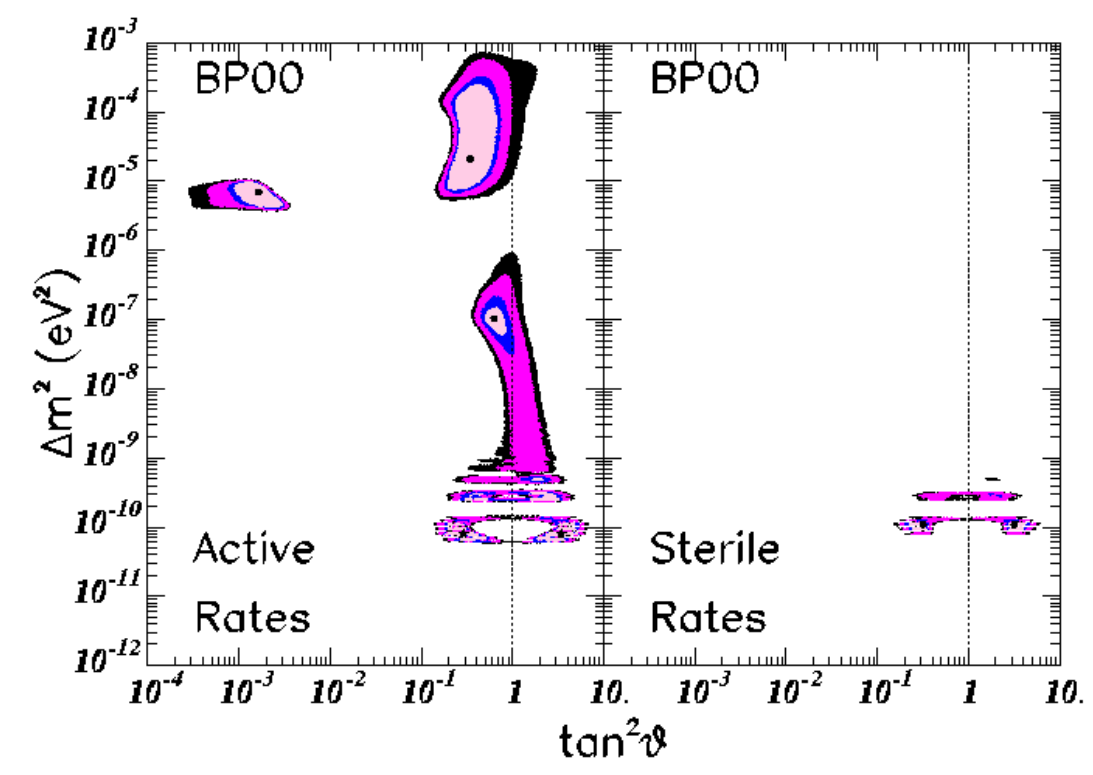

Figure 6: Global solutions for the rates only. The input data are the total rates measured in the SNO CC [1], Chlorine [2], SAGE [⿶] + (GALLEX [3] + GNO [5]), and Super-Kamiokande [6] experiments. The C.L. contours shown in the figure are 90\%, 95\%, $99 \%$, and $99.73 \%(3 \sigma)$. The local best-fit points are marked by dark circles. The theoretical errors for the BP00 neutrino fluxes are included in the analysis.

\begin{tabular}{lcccc}
\hline Solution & $\Delta m^{2}$ & $\tan ^{2}(\theta)$ & $\chi_{\min }^{2}$ & g.o.f. \\
\hline VAC & $7.9 \times 10^{-11}$ & $0.29(3.45)$ & 3.50 & $17 \%$ \\
LMA & $2.1 \times 10^{-5}$ & $3.4 \times 10^{-1}$ & 3.99 & $14 \%$ \\
SMA & $6.9 \times 10^{-6}$ & $1.6 \times 10^{-3}$ & 5.25 & $7 \%$ \\
LOW & $9.7 \times 10^{-8}$ & $6.5 \times 10^{-1}$ & 8.61 & $1.4 \%$ \\
Sterile VAC & $1.1 \times 10^{-10}$ & $0.29(3.45)$ & 10.1 & $0.63 \%$ \\
Sterile SMA & $4.9 \times 10^{-6}$ & $5.3 \times 10^{-4}$ & 18.0 & $0.01 \%$ \\
\hline
\end{tabular}

Table 3: Best-fit parameters for total rates only, corresponding to figure 6. The format of this table is the same as for table 1. The two rates measured by the Gallium experiments, (GALLEX [3] and GNO [5]) and SAGE [4], are included separately. The number of degrees of freedom is 2 [5(rates) -3 (parameters: $\Delta \mathrm{m}^{2}, \theta, O=$ active or sterile neutrino)]. The Sterile SMA solution does not appear in figure 6 because $\chi_{\min }^{2}$ is too large for this solution, but the result is given in the table for general information.

is poorer (cf. table 1 and table 3) when the spectral data are included, which reflects the fact that solutions which predict a flat spectrum in the energy region studied by Super-Kamiokande are favored. 


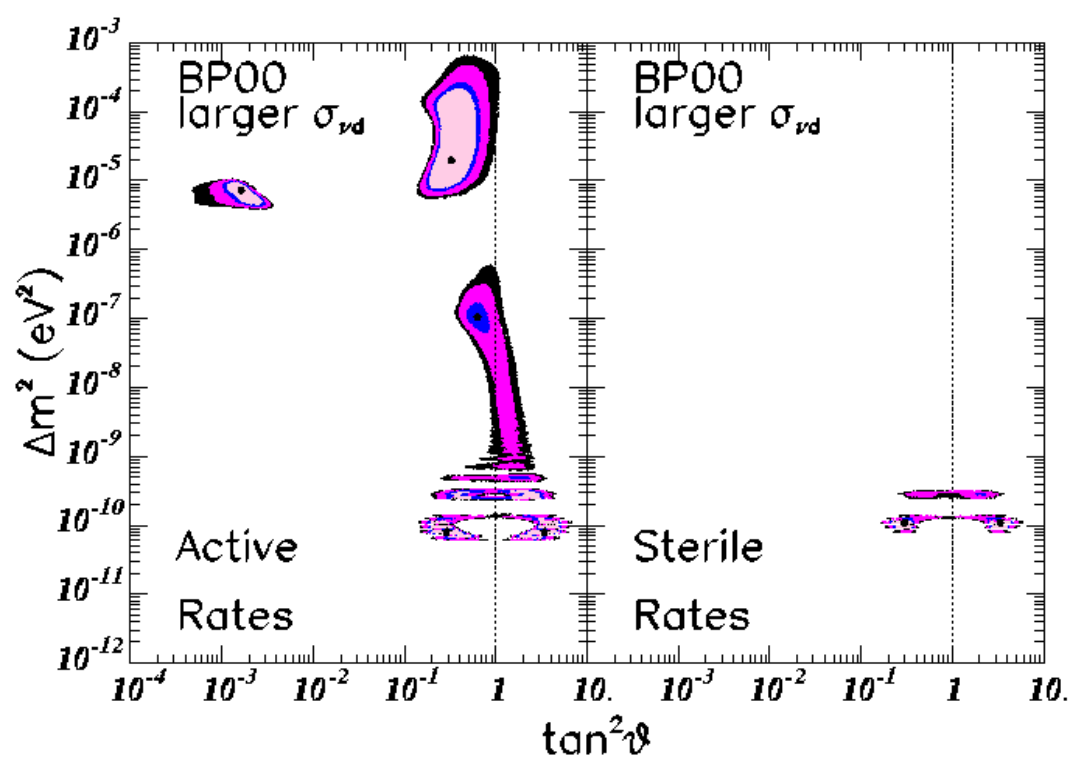

Figure 7: Global solutions for the rates only, with an enhanced CC cross section for deuterium. The input data and the analysis procedures are the same as used in producing figure 6, except that we have used for the present figure a $4 \%$ larger CC cross section for neutrino absorption by deuterium.

The principal changes that result from omitting the spectral and the day-night data are for the vacuum solutions near $10^{-10} \mathrm{eV}^{2}$, the Just $\mathrm{So}^{2}$ solution, and the Sterile SMA solution. The vacuum solutions are much more prominent when the spectral data are not considered. This is a well-know effect and has been noted in many previous analyses. The Just $\mathrm{So}^{2}$ solution is missing when only the rates are considered, which is largely due to the imposition of the BP00 theoretical constraint on the ${ }^{8} \mathrm{~B}$ flux $[8]$. The sterile SMA solution also disappears completely at $3 \sigma$ (just barely, see table 3).

Figure 7 and table 1 show that enhancing the CC cross section by $4 \%$ has very little effect on the allowed regions when only the data on total event rates is considered. This result is seen most clearly by comparing directly figure 6 and table 3 with figure 7 and table $\mathbb{6}$.

Figure 8 shows the allowed solutions that are obtained when the ${ }^{8} \mathrm{~B}$ flux is permitted to vary freely, without considering the constraints implied by the standard solar model. There are no major differences between the allowed regions shown in figure 8 and the allowed regions shown in figure 6 , for which the ${ }^{8} \mathrm{~B}$ neutrino flux was constrained by the BP00 flux predictions.

The minimum $\chi^{2}$ for the rates-only, free ${ }^{8} \mathrm{~B}$ case shown in figure 8 lies in the LMA region, but for a finite value of the active-sterile admixture. The sterile component 


\begin{tabular}{lcccc}
\hline Solution & $\Delta m^{2}$ & $\tan ^{2}(\theta)$ & $\chi_{\min }^{2}$ & g.o.f. \\
\hline VAC & $7.9 \times 10^{-11}$ & $0.29(3.45)$ & 3.07 & $21 \%$ \\
LMA & $2.0 \times 10^{-5}$ & $3.3 \times 10^{-1}$ & 3.45 & $18 \%$ \\
SMA & $7.3 \times 10^{-6}$ & $1.6 \times 10^{-3}$ & 7.09 & $2.9 \%$ \\
Sterile VAC & $1.1 \times 10^{-10}$ & $0.29(3.45)$ & 9.12 & $1.1 \%$ \\
LOW & $1.1 \times 10^{-7}$ & $6.3 \times 10^{-1}$ & 9.45 & $0.89 \%$ \\
Sterile SMA & $4.7 \times 10^{-6}$ & $4.6 \times 10^{-4}$ & 20.7 & $0.003 \%$ \\
\hline
\end{tabular}

Table 4: Best-fit parameters for total rates only with enhanced CC cross section for deuterium, corresponding to figure 7. The input data for this analysis are the same as for the analysis described by table 1 except that a $4 \%$ larger CC cross section for deuterium was used here.

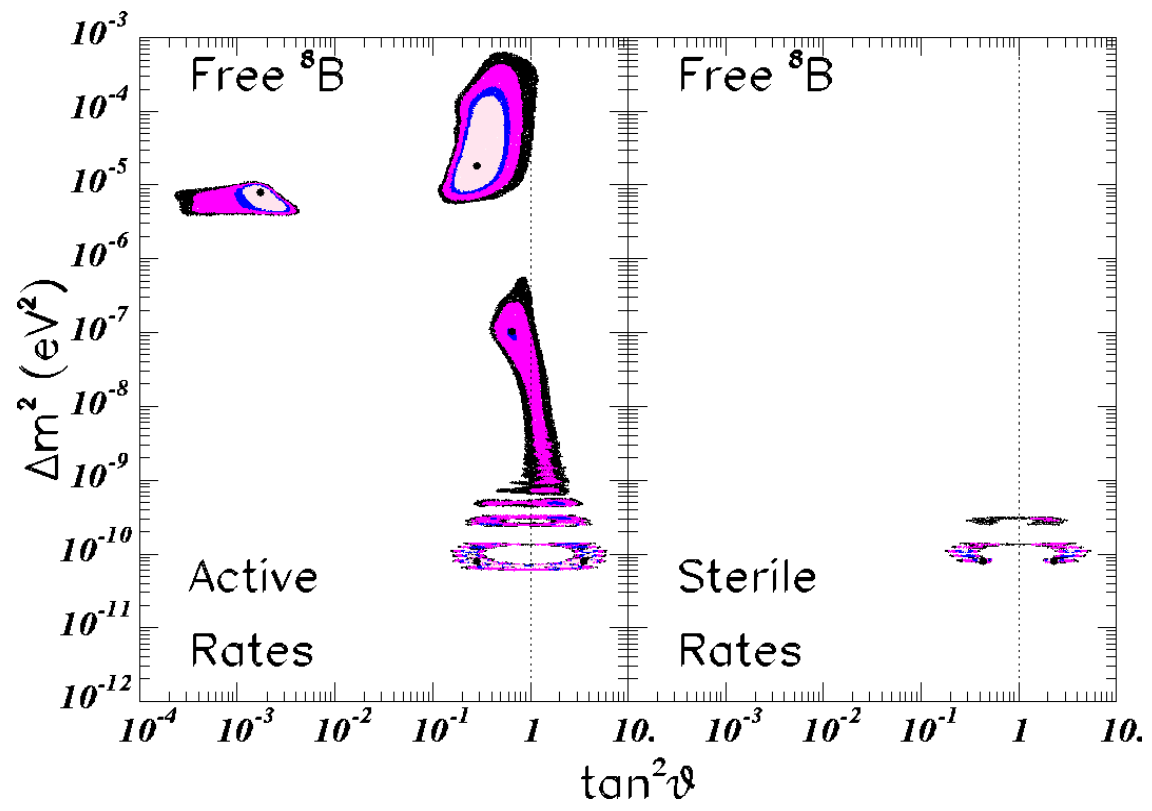

Figure 8: Global solutions for the rates only, with an unconstrained ${ }^{8} \mathbf{B}$ neutrino flux. The input data and the analysis procedures are the same as used in producing figure6, except that the ${ }^{8} \mathrm{~B}$ flux is allowed to vary without considering the predicted flux or errors of the standard solar model.

increases as $f_{\mathrm{B}}$ increases, as described in ref. [24]. We have restricted ourselves to normalization factors $f_{\mathrm{B}} \leq 2$, because (cf. section 2.1) larger values of $f_{\mathrm{B}} \leq 2$ are ruled out at a $3 \sigma$ C.L. by the Super-Kamiokande day-night recoil energy spectrum and are implausible from an astrophysical point of view. With this restriction, the global minimum is found at $\sin ^{2} \eta=0.55$, with $\chi_{\min }^{2}=0.79, \Delta m^{2}=1.3 \times 10^{-5} \mathrm{eV}^{2}$, $\tan ^{2} \theta=0.17$, and $f_{B}=1.9$. The regions shown in figure 8 are defined with respect 
to this global minimum. We have verified that if even larger $f_{\mathrm{B}}$ were allowed the minimum would occur at larger $\sin ^{2} \eta$, in agreement with the results of ref. [24].

We caution, however, that for figure 8 the LMA best-fit point lies in the lower part of the LMA region $\left(\Delta m^{2}=1.8 \times 10^{-5} \mathrm{eV}^{2}\right.$ for pure active neutrinos, $\sin ^{2} \eta=0$, moving down to $\Delta m^{2}=1.2 \times 10^{-5} \mathrm{eV}^{2}$ for $\left.\cos ^{2} \eta=0.55\right)$. This part of the LMA region is disfavored at $3 \sigma$ by the Super-Kamiokande day-night data. For those values of the parameters, the Earth regeneration effects are important and the day-night asymmetry is much larger than observed. Thus we conclude that the results of ref. [24] is correct only the total rates are considered, but cannot be extended to the case in the observed day-night spectral energy data are included. We will return to this point in Sec. 5.3.

Finally, we have investigated the effect of reducing the total error on the $\nu_{e}$ flux measured by SNO. We have supposed, in the same burst of wild optimism that prevailed previously (see section [3, especially the discussion of figure 4 ), that the total error on the $\nu_{e}$ flux is reduced by a factor of three while the best-estimate for the flux remains unchanged from the value quoted by ref. [1]. We find results consistent with the discussion of figure 4 , which applies to the case when all the available solar neutrino data are included in the analysis. In the present case, we find that the SMA solution is greatly reduced in area. The LMA and LOW solutions are not qualitatively affected.

\section{Comparisons and Clarifications}

In this paper, we have done several things that are relatively new in the context of two-component solar neutrino oscillations: *) include the initial SNO CC data [四]; *) implement a prescription first used in ref. [8] for handling consistently the rate normalization and the spectral data for the Super-Kamiokande experiment; and *) treat active and sterile neutrino oscillations on an equal basis [21, 22, 19, 12]. We have already illustrated the effect of the SNO CC measurement by comparing figure 1 with figure 目.

We will clarify in this section the role of the later two aspects of the analysis, the consistent treatment of the rate normalization and the spectral energy distribution, and the level playing field treatment of active and sterile neutrinos. We also compare our results with two recent and very interesting papers, by Barger, Marfatia, and Whisnant 24] and by Fogli, Lisi, Montanino, and Palazzo [27]. In all cases, we reproduce the results of ref. [24] and ref. [27] by analyzing the data in the manner described in these references. The present section was not included in the original version of our paper submitted to the journal, because our analysis was essentially simultaneous with the work of Barger et al. and with Fogli et al.

We begin by discussing in section 5.1 the effect of rejecting a priori the existence of sterile neutrinos and then discuss in section 5.2 the effect of different treatments 
of the measured normalization of the ${ }^{8} \mathrm{~B}$ neutrino flux and the associated influence of the standard solar model constraint on the ${ }^{8} \mathrm{~B}$ neutrino flux. The discussions in section 5.1 and section 5.2 are most relevant to the analysis in ref. [27]. In section 5.3 we show that the small day-night difference measured by Super-Kamiokande disfavors at $3 \sigma$ the primarily sterile neutrino mixtures that were found in ref. [24] when analyzing only the total event rates.

\subsection{A priori rejecting sterile neutrinos}

Assuming that only active neutrinos exist, figure 9 shows the global solutions with and without constraining the ${ }^{8} \mathrm{~B}$ flux by the standard solar model [9]. The two panels in figure 9 should be compared, respectively, with figure 1 and with figure 5 and with figure 6 of ref. [27].

The main effect of rejecting sterile neutrinos a priori is to reduce the area of the allowed regions; the reduction is particularly apparent for the less robust SMA and Just $\mathrm{So}^{2}$ solutions. For the case in which the ${ }^{8} \mathrm{~B}$ neutrinos are constrained by the SSM, both regions become only marginally allowed at $3 \sigma$. For the unconstrained ${ }^{8} \mathrm{~B}$ neutrinos, only the SMA marginally survives at $3 \sigma$ while the Just $\mathrm{So}^{2}$ solution is no longer allowed. This trend is consistent with the results reported in ref. [27].

\subsection{Delicate differences in the error analysis}

A variation on our standard analysis scheme (the results of which are illustrated by figure 1) can teach us a lot about the existence or non-existence of the SMA solution. Depending on the way the normalization of the Super-Kamiokande total rate and the theoretical solar model errors are included in the analysis, the relative quality of the fits can be affected slightly for both the active and the sterile SMA solutions as compared to the fit obtained with the active LMA solution.

If, as done in ref. [27], we include in the analysis the Super-Kamiokande total event rate with its corresponding theoretical error, but allow a free normalization for the Super-Kamiokande day and night energy spectra, (for which no theoretical error is included), we obtain a worse g.o.f. for the SMA solutions while the g.o.f. of the LMA solution is essentially unchanged $\left(\chi_{\mathrm{LMA}}^{2}=35.5, \chi_{\mathrm{SMA}}^{2}=49.8, \chi_{\text {sterile } \mathrm{SMA}}^{2}=\right.$ 53.4). Thus the active and sterile SMA solutions do not appear at the $3 \sigma$ level in such an analysis, which is in agreement with the results of ref. [27].

We trace the origin of the difference in the presence or absence of the SMA

solution to the effect of the "energy independent" ${ }^{8} \mathrm{~B}$ neutrino flux normalization error taken from the standard solar model estimate [9]. As stated above, in ref. [27], the solar model error for ${ }^{8} \mathrm{~B}$ is included for the total Super-Kamiokande rate but not for the free normalization factor in the energy spectrum of the electron recoils. We have included in the present paper the effect of the BP00 errors on the predicted rate in each energy bin of the recoil electron spectrum. When introduced in each of the 

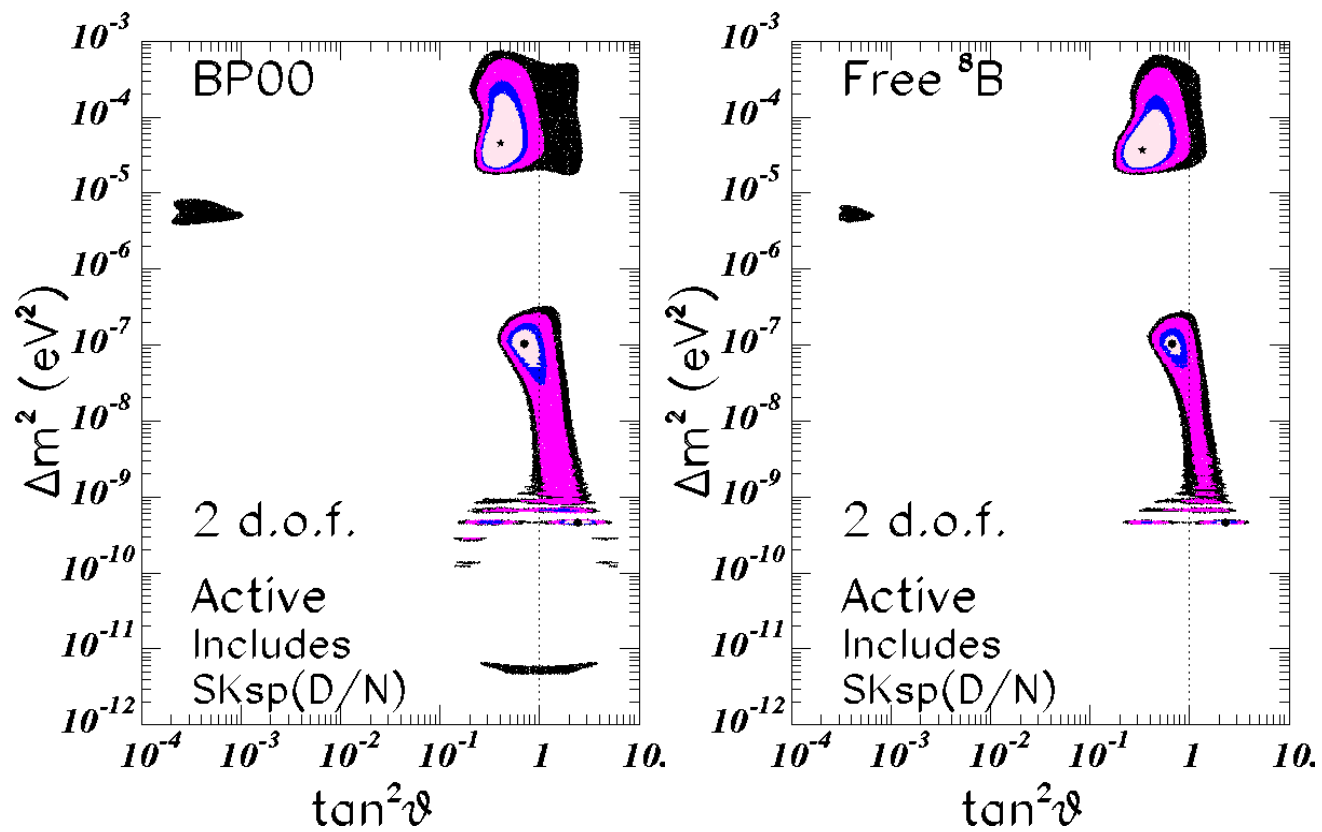

Figure 9: Global solutions assuming a priori that there are only two active neutrinos. The input data and the analysis procedures are the same as used in producing figure 1, except that we have assumed for figure 9 that there are no sterile neutrinos and therefore the analysis is carried out for two d.o.f. . The left and right panels in figure 9 should be compared, respectively, with figure 11 and with figure 5 of this paper and with figure 6 of ref. [27].

spectrum bins, this theoretical error in the total flux produces an effectively energydependent error if the survival probability depends upon energy and our procedure is therefore not equivalent to considering the theoretical error to apply only to the total rate. In other words, if the expected number of events is different in each energy bin, the theoretical error of this expected number of events is different in each energy bin, even if the relative error is energy independent. As a consequence, shifting up or down the theoretical prediction within the $1 \sigma$ theoretical error, results also in a change of the predicted spectrum shape. In particular, for a given point in the SMA region, moving $1 \sigma$ down within the theoretical errors (needed to fit better the observed number of events in Super-Kamiokande and SNO) results in a flatter predicted electron energy spectrum (less distortion due to oscillations) and fits better the observed Super-Kamiokande recoil electron energy spectrum.

Including the ${ }^{8} \mathrm{~B}$ neutrino flux normalization error in the predicted rate in each energy bin of the recoil electron energy spectrum leads to a better fit for the Just $\mathrm{So}^{2}$ solution than when the analysis is performed with the theoretical error included only in the total Super-Kamiokande rate (as in ref. [27]). For the Just $\mathrm{So}^{2}$ solution, the expected rate is larger than the measured value at Super-Kamiokande, so the 
$20 \%$ theoretical ${ }^{8} \mathrm{~B}$ flux normalization error is larger for the Just $\mathrm{So}^{2}$ solution than the corresponding error for the LMA solution. The larger error for Just $\mathrm{So}^{2}$, when added to the 38 spectrum data points, even if fully correlated, leads to a relatively lower $\chi^{2}$ and also to a smaller impact of the SNO CC measurement on the quality of the Just $\mathrm{So}^{2}$ solution for the global fit. Consistent with this explanation the Just $\mathrm{So}^{2}$ solution does not appear when the analysis is performed with the total rates only (see, e. g., Figure 6).

We have further verified that when the analysis is performed with unconstrained ${ }^{8} \mathrm{~B}$ fluxes and therefore no theoretical error for the ${ }^{8} \mathrm{~B}$ flux is included, both approaches, either including the Super-Kamiokande total event rate and a free spectrum normalization, or not including the Super-Kamiokande total rate and using the spectrum normalization information, lead to very similar results. Thus the results shown in figure 5 hold for both of the above-described analysis variations.

Thus the presence or absence of the SMA solution at $3 \sigma$ depends upon whether and how the standard solar model errors on the ${ }^{8} \mathrm{~B}$ neutrino flux are introduced into the analysis.

The question of whether or not the SMA solution is ruled out at $3 \sigma$ is an example where we think it is useful to apply the motto: "If it is not robust, it is not believable."

\subsection{Mixtures of sterile and active neutrinos}

We summarize in this subsection our method and results involving mixtures of sterile and active neutrinos. We pay particular attention to the case in which the ${ }^{8} \mathrm{~B}$ neutrino flux is unconstrained. For this case, we compare our results with those of Barger, Marfatia, and Whisnant, ref. [24].

In order to "level the playing field", we treat active and sterile neutrinos as different aspects of the same two-neutrino oscillation scheme. Assuming that either all solar neutrinos oscillate into all active or all sterile neutrinos corresponds to the limiting extremes of a continuum in which the oscillation occurs into a linear combination of active and sterile neutrinos, cf. discussion of four neutrino oscillations in ref. 21]. We use the available experimental data to determine the favored composition of the final state. In the different figures in this paper, we show the allowed solution regions in the limiting extremes in which the neutrino state into which $\nu_{e}$ 's oscillate is purely active neutrinos or purely sterile neutrinos. We minimize $\chi^{2}$ in 3 -dimensional parameter space and define the allowed regions with respect to the global minimum in terms of shifts of $\Delta \chi^{2}$ for 3 d.o.f. (cf. discussion in section 2.1).

For the analysis performed using the standard solar model fluxes and errors, we find that the global best-fit point lies within the LMA allowed region for purely active neutrinos. This result holds for both the global analysis using all available solar neutrino data as well as for the analysis that includes only the information on the total rates. 
For the analysis performed for unconstrained ${ }^{8} \mathrm{~B}$ neutrinos, the situation is somewhat complex. Barger and his colleagues, see ref. [24], have pointed out that even after the SNO CC result one can obtain a good fit for primarily oscillations into sterile neutrinos if the ${ }^{8} \mathrm{~B}$ solar neutrino flux is allowed to be considerably larger than the BP00 prediction.

We confirm the conclusion of Barger et al. on the viability of primarily sterile neutrino oscillation solutions provided that the analysis is performed using only the information on the total event rates. In this case, the best fit to the rate data lies in the LMA region but with a non-zero sterile neutrino component that increases in amplitude as one increases the assumed ${ }^{8} \mathrm{~B}$ neutrino normalization factor, $f_{\mathrm{B}}$ (for the definition of $f_{\mathrm{B}}$ see section 2.1).

However, once the Super-Kamiokande day-night spectral energy data are included in the analysis, we find that the best fit corresponds to purely active os-

cillations (zero sterile neutrino component). The small day-night effect measured by Super-Kamiokande is the reason for this difference between the potentially large sterile component that can be present in the rates-only analysis and the depressed sterile component that is present in the global analysis. The solutions with a large sterile component imply a large day-night effect in Super-Kamiokande, which was not measured. Thus we conclude that the results of ref. 24] on the continued viability of a large sterile solar neutrino flux, although correct for the analysis of only the total rates, do not apply once the day-night spectral data are included.

\section{Discussion}

The theme of this paper is that robust conclusions regarding solar neutrino oscillation parameters are independent of plausible variations in the analysis strategy. Different groups have explored slightly different strategies and we have considered and compared in this paper a variety of possible approaches. We show, for example, that some conclusions depend upon the specific way that the theoretical uncertainties are included in the analysis. We advocate accepting as established only those inferences that do not depend sensitively upon the details of how the analysis is performed.

Our principal conclusions are simple to state.

First, for every one of the analysis strategies used in this paper, the favored solutions all involve large mixing angles: LMA, LOW, or VAC. The MSW solutions are favored over the vacuum solutions if all of the data, not just the total rates, are considered.

Second, all eight of the previously recognized two component oscillation solutions are still allowed at $3 \sigma$ if one considers in the analysis the solar model constraints on the ${ }^{8} \mathrm{~B}$ neutrino flux and all the available neutrino data, including the SNO CC measurement and the Super-Kamiokande spectral energy distributions in the day and at night. The goodness of fit for all of these solutions ranges from good to satisfactory. 
The results are shown in figure 1 and table 1, which describe the currently preferred global solution.

Third, our results agree in broad outline with the recent contemporary analyses of the solar neutrino data presented in refs. [24, 27]. The small differences between the separate analyses depend in physically understandable ways upon different strategies and assumptions that have been adopted, as discussed in section 5 .

Fourth, even the extreme assumption of ignoring all the Super-Kamiokande data on the spectral recoil energy distribution and the day-night variations does not change very much the allowed regions of the preferred solutions. Only the marginally allowed Just $\mathrm{So}^{2}$ active and sterile solutions and the SMA sterile solution are not allowed at $3 \sigma$ when the spectral and day-night data are ignored. This result can be seen by comparing figure 6 with figure 1 .

Fifth, the oscillation scenarios with a very large sterile neutrino component that were found in ref. [24] when analyzing only the total event rates are disfavored at $3 \sigma$ when the day-night spectral energy distribution measured by Super-Kamiokande is included in the analysis(see section 5.3).

Sixth, the global solutions that are obtained with and without solar model constraints on the ${ }^{8} \mathrm{~B}$ neutrino flux are very similar for the favored large mixing angle solutions. This result can be seen by comparing visually figure 1 and figure 5 (both constructed using all the available solar neutrino data) and figure 6 and figure 8 (both constructed using only the data on the total rates). The only apparent differences occur for marginally allowed regions like the sterile solutions and the active Just $\mathrm{So}^{2}$ solution. The reason for the insensitivity to how the ${ }^{8} \mathrm{~B}$ neutrino flux is treated is that the combined SNO and Super-Kamiokande measurement of the total flux [1] is in excellent agreement with the standard solar model prediction [9].

Seventh, the allowed solution space is not qualitatively affected by a suggested [10] increase by $4 \%$ of the CC cross section on deuterium, although the allowed regions of the SMA and the Just $\mathrm{So}^{2}$ solutions become somewhat smaller when the cross section is enhanced. This result can be inferred from a comparison of figure 1 and figure 3 .

Eighth, we have investigated the effect of reducing the total error by a factor of three on the experimental measurement of the $\nu_{e}$ flux while the best-estimate flux remains constant at the value quoted in ref. [1]. The currently allowed LMA and SMA solutions are not much affected by this hypothetical and optimistic error reduction, but the SMA is eliminated at $3 \sigma$ from the global solution with all the data included, as are also the active and sterile Just $\mathrm{So}^{2}$ solutions. These results are apparent when comparing figure 1 with figure 1.

In summary, the CC measurement by SNO has not changed qualitatively the globally allowed solution space for solar neutrinos, although the CC measurement has provided dramatic and convincing evidence for neutrino oscillations and has strengthened the case for active oscillations with large mixing angles. These results are robust 
and does not depend sensitively on the details of the analysis assumptions. Future SNO measurements [28], including the day-night effect, the spectral energy distribution, and the neutral current to charge current ratio, will significantly reduce the allowed regions of parameter space [14]. The KamLAND [29] and BOREXINO [30] experiments will provide stringent diagnostics of different oscillation scenarios.

We are grateful to a number of colleagues for insightful remarks and stimulating questions regarding an earlier draft of this paper. We especially appreciate comments by J. Beacom, M. Chen, R. Gandhi, P. Krastev, E. Lisi, A. McDonald, S. Petcov, H. Robertson, and G. Steigman. JNB acknowledges support from NSF grant No. PHY0070928. MCG-G thanks the School of Natural Sciences in the Institute for Advanced Study (Princeton), where part of this work was carried out, for warm hospitality. CPG thanks the CERN theory division for their hospitality. MCG-G is supported by the European Union Marie-Curie fellowship HPMF-CT-2000-00516. This work was also supported by the Spanish DGICYT under grants PB98-0693 and PB97-1261, by the Generalitat Valenciana under grant GV99-3-1-01, and by the TMR network grant ERBFMRXCT960090 of the European Union and ESF network 86. 


\section{References}

[1] Q.R. Ahmad, et al., Measurement of charged current interactions produced by ${ }^{8} \mathrm{~B}$ solar neutrinos at the Sudbury Neutrino Observatory, submitted to Phys. Rev. Lett..

[2] B.T. Cleveland et al., Measurement of the solar electron neutrino flux with the Homestake chlorine detector, Astrophys. J. 496 (1998) 505.

[3] W. Hampel et al. (GALLEX Collaboration), GALLEX solar neutrino observations: results for GALLEX IV, Phys. Lett. B 447 (1999) 127.

[4] J.N. Abdurashitov et al. (SAGE Collaboration), Measurement of the solar neutrino capture rate with gallium metal, Phys. Rev. C 60 (1999) 055801 astro-ph/9907113; V. Gavrin (SAGE Collaboration), Solar neutrino results from SAGE, in Neutrino 2000, Proc. of the XIXth International Conference on Neutrino Physics and Astrophysics, 16-21 June 2000, eds. J. Law, R.W. Ollerhead, and J.J. Simpson, Nucl. Phys. Proc. Suppl. B 91 (2001) 36.

[5] M. Altmann et al. (GNO Collaboration), GNO solar neutrino observations: results for GNO I, Phys. Lett. B 490 (2000) 16; E. Bellotti et al. (GNO Collaboration), First results from GNO, in Neutrino 2000, Proc. of the XIXth International Conference on Neutrino Physics and Astrophysics, 16-21 June 2000, eds. J. Law, R.W. Ollerhead, and J.J. Simpson, Nucl. Phys. Proc. Suppl. B 91 (2001) 44.

[6] Y. Fukuda et al. (Super-Kamiokande Collaboration), Measurements of the solar neutrino flux from Super-Kamiokande's first 300 days, Phys. Rev. Lett. 81 (1998) 1158; Erratum 81 (1998) 4279; Constraints on neutrino oscillation parameters from the measurement of day-night solar neutrino fluxes at Super-Kamiokande, Phys. Rev. Lett. 82 (1999) 1810; Y. Suzuki (Super-Kamiokande Collaboration), Solar neutrino results from Super-Kamiokande, in Neutrino 2000, Proc. of the XIXth International Conference on Neutrino Physics and Astrophysics, 16-21 June 2000, eds. J. Law, R.W. Ollerhead, and J.J. Simpson, Nucl. Phys. Proc. Suppl. B 91 (2001) 29; S. Fukuda et al. (Super-Kamiokande Collaboration), "Solar ${ }^{8} \mathrm{~B}$ and hep neutrino measurements from 1258 days of Super-Kamiokande data," Phys. Rev. Lett. 86 (2001) 5651.

[7] P. Creminelli, G. Signorelli and A. Strumia, Frequentist analyses of solar neutrino data, JHEP 0105, 052 (2001) hep-ph/0102234.

[8] J.N. Bahcall, P.I. Krastev and A.Yu. Smirnov, Solar neutrinos: global analysis and implications for SNO, JHEP 0105(2001)015 hep-ph/0103179.

[9] J.N. Bahcall, M.H. Pinsonneault and S. Basu, Solar models: current epoch and time dependences, neutrinos, and helioseismological properties, Astrophys. J. $\mathbf{5 5 5}$ (2001)(to be published) astro-ph/0010346.

[10] J.F. Beacom and S.J. Parke, On the normalization of the neutrino-deuteron cross section hep-ph/0106128]. 
[11] M. C. Gonzalez-Garcia, M. Maltoni, C. Peña-Garay and J.W.F. Valle, Global threeneutrino oscillation analysis of neutrino data, Phys. Rev. D 63 (2001) 033005 hep$\mathrm{ph} / 0009350$.

[12] G.L. Fogli and E. Lisi, Standard solar model uncertainties and their correlations in the analysis of the solar neutrino problem, Astropart. Phys. 3 (1995) 185 with updated uncertainties as discussed in G.L. Fogli, E. Lisi, D. Montanino and A. Palazzo, Threeflavor MSW solutions of the solar neutrino problem, Phys. Rev. D 62 (2000) 013002.

[13] M.C. Gonzalez-Garcia and C. Peña-Garay, Four-neutrino oscillations at SNO, Phys. Rev. D 63 (2001) 073013.

[14] J.N. Bahcall, P.I. Krastev and A.Yu. Smirnov, SNO: predictions for ten measurable quantities, Phys. Rev. D 62 (2000) 93004.

[15] S. Nakamura, T. Sato, V. Gudkov and K. Kubodera, Neutrino reactions on the deuteron, Phys. Rev. C 63 (2001) 034617.

[16] M. Butler, J.-W. Chen and X. Kong, Neutrino-deuteron scattering in effective field theory at next-to-next-to-leading order, Phys. Rev. C 63 (2001) 035501.

[17] I.S. Towner, Radiative corrections in neutrino-deuterium scattering, Phys. Rev. C 58 (1998) 1288.

[18] A. McDonald and H. Robertson, private communications.

[19] G.L. Fogli, E. Lisi and D. Montanino, Matter-enhanced three-flavor oscillations and the solar neutrino problem, Phys. Rev. D 54 (1996) 2048.

[20] A. Gouvea, A. Friedland and H. Murayama, The dark side of the solar neutrino parameter space, Phys. Lett. B 490 (2000) 125.

[21] D. Dooling, C. Giunti, K. Kang and C.W. Kim, Matter effects in four-neutrino mixing, Phys. Rev. D 61 (2000) 073011; C. Giunti, M.C. Gonzalez-Garcia and C. Peña-Garay, Four-neutrino oscillation solutions of the solar neutrino problem, Phys. Rev. D 62 (2000) 013005.

[22] D. Harley, T. K. Kuo and J. Pantaleone, Phys. Rev. D 47 (1993) 4059

[23] M. Apollonio et al., Limits on neutrino oscillations from the CHOOZ experiment,Phys. Lett. B 466 (1999) 415.

[24] V. Barger, D. Marfatia and K. Whisnant, 'Unknowns after the SNO charged-current measurement, hep-ph/0106207]; V. Barger, D. Marfatia and K. Whisnant, Piecing the Solar Neutrino Puzzle Together at SNO, Phys. Lett. B509 (2001) 19-29.

[25] R.S. Raghavan, Solar neutrinos - from puzzle to paradox, Science 267 (1995) 45.

[26] P.I. Krastev and S.T. Petcov, On the vacuum oscillation solutions of the solar neutrino problem, Phys. Rev. D 53 (1996) 1665. 
[27] G.L. Fogli, E. Lisi, D. Montanino and A. Palazzo, Model-dependent and independent implications of the first Sudbury Neutrino Observatory results, hep-ph/0106247.]

[28] A.B. McDonald (SNO collaboration), The Sudbury Neutrino Observatory project, Nucl. Phys. B 77 (Proc. Suppl.) (1999) 43; G.T. Ewan, W.F. Davidson and C.K. Hargrove (SNO Collaboration), The Sudbury Neutrino Observatory - an introduction, Physics in Canada 48 (1992) 112; SNO Collaboration, The Sudbury Neutrino Observatory, Nucl. Instrum. Meth. A 449 (2000) 172.

[29] P. Alivisatos et al. (the KamLAND collaboration), The KamLAND proposal, Stanford-HEP-98-03; A. Piepke (KamLAND Collaboration), KamLAND: A reactor neutrino experiment testing the solar neutrino anomaly, in Neutrino 2000, Proc. of the XIXth International Conference on Neutrino Physics and Astrophysics, 16-21 June 2000, eds. J. Law, R.W. Ollerhead and J.J. Simpson, Nucl. Phys. B 91 (Proc. Suppl.), 99.

[30] G. Alimonti et al. (BOREXINO collaboration), Science and Technology of BOREXINO: a real time detector for low energy solar neutrinos [hep-ex/0012030]. 